\title{
Rare genetic causes of meningitis and encephalitis
}

\author{
Jangsup Moon \\ Rare Disease Center, Departments of Genomic Medicine and Neurology, Seoul National University Hospital, Seoul, Korea
}

Differential diagnosis of meningitis and encephalitis is often very challenging because it cannot be determined based on symptoms, and the diseases have various causes. This article explains rare genetic causes of meningitis and encephalitis. Autoinflammatory disorders include cryopyrin-associated periodic syndromes, familial Mediterranean fever, and tumor necrosis factor receptor-associated periodic syndrome. Furthermore, other genetic disorders, such as complement factor I deficiency, phosphatidylinositol glycan anchor biosynthesis class T mutation, and neuronal intranuclear inclusion disease, can present as meningitis and encephalitis.

Keywords: Rare diseases, Inborn genetic diseases, Meningitis, Encephalitis, Differential diagnosis

\section{Introduction}

Diagnosis and management of meningitis and encephalitis are challenging for clinicians because of the variety of causes [1]. Over the past decade, autoimmune encephalitis (AE) has become more recognized, and its reported incidence has increased. AE can be diagnosed based on clinical diagnostic criteria regardless of the presence of autoantibodies [2,3]. The existence of autoantibodies should not be a prerequisite for AE diagnosis because not all disease-causing antibodies have been identified, with novel antibodies discovered in AE patients every year [4-6]. Due to the increased awareness of AE and its potential responsiveness to immunotherapy, antibody-negative AE currently occupies a substantial portion of AE. Though immunotherapy is performed in patients with antibody-negative AE, the response varies [7-9]. In suspected AE cases with insufficient treatment response, the possibility of other diagnoses should be considered.

In recent years, genetic diagnosis of rare diseases has been increasing due to advances in sequencing technology $[10,11]$. Consequently, rare genetic diseases that occur in the form of meningitis or encephalitis have been discovered. This study summarizes the types of rare genetic diseases that should be considered as differential diagnosis of meningitis and encephalitis (Table 1).

\section{Autoinflammatory disorders}

Autoinflammatory disorders (AIDs) are systemic disorders of innate immunity without the presence of antibodies or reactive T cells [12]. AIDs usually manifest during childhood and are characterized by repeated episodes of inflammation; however, adult-onset cases have been reported [13]. Aseptic meningitis can be an initial manifestation of AIDs, complicating its distinction from autoimmune or infectious diseases [14]. Subtypes of AIDs that can present as aseptic meningitis are discussed in the following section (Table 1).

\section{Cryopyrin-associated periodic syndromes: NLRP3 mutation}

Cryopyrin-associated periodic syndromes (CAPS) are a group of autosomal dominant hereditary systemic autoinflammatory diseases that include mild subtype familial cold-induced

Received: September 21, 2021 Revised: December 15, 2021 Accepted: December 24, 2021

Correspondence: Jangsup Moon

Departments of Genomic Medicine and Neurology, Seoul National University Hospital, 101 Daehak-ro, Jongno-gu, Seoul 03080, Korea

E-mail: jangsup.moon@gmail.com

ORCID: https://orcid.org/0000-0003-1282-4528

Copyright (C) 2022 by The Korean Encephalitis and Neuroinflammation Society

This is an open access article distributed under the terms of the Creative Commons Attribution Non-Commercial License (http://creativecommons.org/licenses/by-nc/4.0/) which permits unrestricted non-commercial use, distribution, and reproduction in any medium, provided the original work is properly cited. 
Table 1 Summary of monogenic disorders that can cause meningitis and encephalitis

\begin{tabular}{|c|c|c|c|c|c|}
\hline Disease & Gene & Inheritance & Age of onset (yr) & Neurological symptom & Systemic symptom \\
\hline CAPS & NLRP3/CIAS1 & $A D$ & Variable & Aseptic meningitis & $\begin{array}{l}\text { Fever, urticaria-like rash, conjunctivitis, } \\
\text { arthralgia, sensorineural hearing loss }\end{array}$ \\
\hline FMF & MEFV & $\mathrm{AR} / \mathrm{AD}^{\mathrm{a}}$ & $0-30$ & Aseptic meningitis & Fever, rash, serositis, arthritis (in large joints) \\
\hline TRAPS & TNFRSF1A & $A D$ & Variable & Aseptic meningitis & $\begin{array}{l}\text { Fever, migrating skin rash, serositis, periorbital } \\
\text { edema, arthritis }\end{array}$ \\
\hline CFI deficiency & CFI & AR & $0-60$ & $\begin{array}{l}\text { Aseptic meningitis, hemorrhagic } \\
\text { leukoencephalitis }\end{array}$ & $\begin{array}{l}\text { Severe recurrent infections, glomerulonephritis, } \\
\text { pneumonia, arthritis }\end{array}$ \\
\hline PIGT mutation & PIGT & AR & Variable (neonatal-adult) & $\begin{array}{l}\text { Aseptic meningitis, neonatal } \\
\text { seizure, intellectual disability }\end{array}$ & $\begin{array}{l}\text { Fever, paroxysmal nocturnal hemoglobinuria, } \\
\text { urticaria, arthralgia }\end{array}$ \\
\hline NIID & NOTCH2NLC & $A D$ & Variable $(<40)$ & $\begin{array}{l}\text { Dementia, cerebellar ataxia, } \\
\text { parkinsonism, peripheral } \\
\text { neuropathy, seizure, pupil } \\
\text { constriction }\end{array}$ & $\begin{array}{l}\text { Chronic cough, paroxysmal chest pain, postural } \\
\text { hypotension, muscle weakness, joint pain, } \\
\text { urinary frequency/urgency, constipation }\end{array}$ \\
\hline
\end{tabular}

CAPS, cryopyrin-associated periodic syndromes; FMF, familial Mediterranean fever; TRAPS, tumor necrosis factor receptor-associated periodic syndrome; CFI, complement factor I; PIGT, phosphatidylinositol glycan anchor biosynthesis class T; NIID, neuronal intranuclear inclusion disease; AD, autosomal dominant; AR, autosomal recessive. ${ }^{a} \mathrm{FMF}$ is classically known as AR disease; however, rare cases of AD FMF with specific heterozygous mutations have been reported.

autoinflammatory syndrome (FCAS), Muckle-Wells syndrome (MWS), and chronic infantile neurological, cutaneous, and articular syndrome (CINCA; also called neonatal-onset multisystem inflammatory disease), which is the most severe subtype $[15,16]$. The syndromes are all caused by heterozygous gain-of-function mutation in the NLRP3 gene (also known as CIAS1, NALP3, or PYPAF1) that encodes the protein cryopyrin, a component of the NLRP3 inflammasome. This mutation leads to hyperactivation of the inflammasome and subsequent overproduction of proinflammatory cytokine interleukin (IL)- $1 \beta$ and inflammatory symptoms. The diagnosis of CAPS is challenging due to the heterogeneous multisystemic clinical presentation, somatic mosaicism, and low-penetrance mutations $[16,17]$.

Historically, FCAS, MWS, and CINCA have been accepted as three distinct diseases but are now considered a single disease spectrum with overlapping signs and symptoms. CAPS are multisystem inflammatory diseases that affect skin, muscles, joints, bones, eyes, ears, and the central nervous system (CNS) [16]. CNS involvement is the most devastating feature of CAPS. Although patients with MWS rarely have intermittent aseptic meningitis, patients with CINCA often develop recurrent chronic aseptic meningitis leading to increased intracranial pressure, hydrocephalus, papilledema, headache, and seizures [15].

Other typical signs and symptoms of CAPS include dermatological (urticarial-like rash), musculoskeletal (arthralgia, arthritis, myalgia, limb-length discrepancies and contractures, clubbing, patellar overgrowth), ocular (conjunctivitis, uveitis, papilledema, progressive amaurosis), and inner ear (sensori- neural hearing loss) manifestations [18]. However, fever is not a common presenting symptom in patients with CAPS, and body temperature does not meet the standard criteria for fever [16].

By 2018, more than 90 confirmed disease-causing mutations in NLRP3 have been reported. More than $90 \%$ of these mutations are located in exon 3 , which encodes the central regulatory domain of the protein and is reported to show good genotype-phenotype correlation, indicating that each mutation is likely associated with only one clinical phenotype among the disease continuum. Up to $50 \%$ of patients with CINCA, $25 \%$ of patients with MWS, and $10 \%$ of patients with FCAS do not have a germline NLRP3 mutation [19], and the majority of these patients have somatic mosaicism in NLRP3 [16]. Low-penetrance mutations have been reported in CAPS, indicating that only a small portion of subjects carrying the mutation will develop symptoms. In particular, V198M, R488K, and Q703K variants have been described in up to $10 \%$ of the normal population $[16,20]$.

Diagnosis of CAPS can be made clinically and genetically but is often delayed. Low awareness of CAPS among physicians and cases with somatic mutations are the main cause of delay in diagnosis. Although early onset of symptoms is a strong indicator for CAPS, some patients show only mild symptoms, indicating the importance of consideration of CAPS in adults [21]. Clinical diagnostic criteria for CAPS have been recently developed [22]. Increased inflammatory markers are a mandatory criterion, and more than two of six additional items are required for diagnosis (Figure 1). The presence of $N L R P 3 \mathrm{mu}$ tations can be confirmatory although not necessary for diag- 
Figure 1 Diagnostic criteria for CAPS

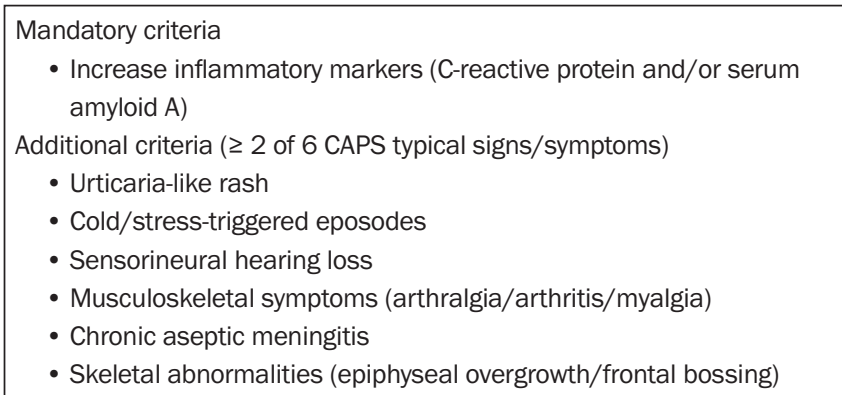

CAPS, cryopyrin-associated periodic syndromes.

nosis of CAPS; thus, a genetic diagnosis is required before initiation of appropriate therapy.

Despite clinical heterogeneity, patients with CAPS respond well to IL-1 blockade treatment. To date, three IL-1 blockade therapies, anakinra (Kineret; Sobi, Stockholm, Sweden), rilonacept (Arcalyst; Regeneron, New York, NY, USA), and canakinumab (Ilaris; Novartis, Basel, Switzerland), have been approved by the Food and Drug Administration or European Medicines Agency for treatment of CAPS [16].

\section{Familial Mediterranean fever}

Familial Mediterranean fever (FMF) is the most common monogenic cause of AID and is characterized by recurrent fever and multiple serositis. FMF is an autosomal recessive disorder caused by mutations in the $M E F V$ gene, which encodes the protein pyrin. Pyrin negatively regulates the activation of inflammasomes through regulation of IL-1 $\beta$ production [23]. Pyrin mutations lead to increased synthesis of proinflammatory cytokines (IL-1 $\beta$ and IL-6) and cause changes in inflammasome function. Although FMF is typically inherited in an autosomal recessive manner, dominant inheritance with specific heterozygous mutations has been reported [24].

Disease onset occurs before 10 years of age in more than $60 \%$ of patients and before 30 years of age in $98 \%$ of cases [25]. Adult-onset patients often have milder phenotype and can present with uncommon manifestations such as aseptic meningitis. Aseptic meningitis in FMF follows a similar pattern, with typical symptoms including self-limiting episodes lasting 3 to 5 days. However, according to previous reports, aseptic meningitis is a very rare manifestation of FMF. In one study, only seven of 12,000 adult patients with FMF between 1985 and 2010 experienced aseptic meningitis without a clear de- scription of recurrent attacks [26]. During the last 10 years, five cases of recurrent aseptic meningitis have been reported [27]. The age of onset ranged from 5 to 46 years, and every patient responded to colchicine treatment. Notably, all five patients carried the E148Q variant in exon 2 as homozygote or heterozygote accompanied by another variant. None of the patients had a pathogenic variant in exon 10.

To date, more than $370 \mathrm{MEFV}$ nucleotide variants have been reported. Among them, five variants, M694V, M694I, V726A, and M680I in exon 10, and E148Q in exon 2, are responsible for approximately $85 \%$ of cases [28]. The majority of FMF-causing mutations are located in $M E F V$ exon 10 and associated with the typical and severe phenotype of recurrent inflammatory episodes. The typical FMF phenotype includes periodic fever and abdominal or chest pain lasting for a few hours to 3 days. Notably, M694del has been reportedly associated with autosomal dominantly inherited FMF [24,29].

In terms of treatment, colchicine is considered the gold standard for FMF patients. When colchicine treatment is ineffective, other biologics such as IL-1 inhibitors and tumor necrosis factor (TNF)- $\alpha$ blockers can be used. Examples include anakinra, canakinumab, and rilonacept as IL-1 inhibitors and infliximab and etanercept as TNF- $\alpha$ blockers [30].

\section{Tumor necrosis factor receptor-associated periodic syndrome}

TNF receptor-associated periodic syndrome (TRAPS) is an autosomal dominant disorder caused by mutation in the $T N$ FRSF1A gene that encodes TNF-receptor 1. Among the monogenic AIDs, TRAPS shows the most variable manifestation in terms of age of onset, disease duration, and clinical presentation. The pathogenesis of TRAPS is complex and involves multiple mechanisms including increased endoplasmic reticulum stress, excessive mitochondrial reactive oxygen species, and enhanced nuclear factor kappa B (NF- $\mathrm{BB}$ ) activation [31].

Most TRAPS patients experience early-onset symptoms, but adult-onset TRAPS has been widely reported, especially in subjects carrying low-penetrance mutations and presenting an atypical phenotype. Fever and serositis presenting as migratory skin rash, myalgia, abdominal pain, and periorbital edema are the most common clinical features [31]. Aseptic meningitis is considered an atypical symptom of TRAPS, and CNS involvement has been reported in a limited number of cases [32,33]. 
To date, no clinical diagnostic criteria of TRAPS have been proposed; thus, diagnosis of TRAPS is based on identification of TNFRSF1A mutation. All TRAPS pathogenic variants are clustered in exons 2-4; however, R92Q and P46L are considered low-penetrance variants [31].

Corticosteroids are very effective for treatment of TRAPS, but chronic use is limited due to their long-term toxicity. IL-1 blocking agents are suggested as treatment of choice for the majority of patients with active TRAPS [31].

\section{Other genetic disorders}

In addition to the above-mentioned AIDs, several other monogenic diseases resemble meningitis and encephalitis. Therefore, although very rare, the possibility of the following genetic disorders in patients with meningitis and encephalitis should be considered.

\section{Complement factor I deficiency}

Defect in homeostasis of the complement system can result in autoimmune reactions with variable clinical presentations. Complement factor I (CFI) is a serine protease with an important regulatory role in the alternative complement pathway. CFI deficiency, a rare autosomal recessive disorder, is associated with recurrent infections as well as relapsing inflammatory responses.

Haerynck et al. [34] reported a case of a 16-year-old female with CFI deficiency who experienced recurrent episodes of acute aseptic meningoencephalitis. Ten episodes of meningoencephalitis occurred over a 15-month period. Corticosteroid treatment and plasmapheresis were effective, but she relapsed several times after tapering steroids. Cyclophosphamide and azathioprine were administered to reduce the daily dose of steroids, severe relapses prompted a change to mycophenolate mofetil, and no further relapse occurred.

Broderick et al. [35] described two unrelated pediatric patients with acute hemorrhagic leukoencephalitis caused by CFI deficiency. Both patients showed extensive white matter signal abnormalities on magnetic resonance imaging (MRI), and brain biopsy revealed neutrophilic inflammation and patchy demyelination. Administration of corticosteroid with intravenous immunoglobin and anakinra (IL-1 receptor antagonist) helped improve the white matter lesions and the clinical symptoms.
To date, only about 25 cases of genetically confirmed CFI deficiency have been reported. At the time of diagnosis, patient age ranged from infancy to 60 years, and clinical symptoms varied widely from severe bacterial infections to recurrent sterile neuroinflammations [36-38].

\section{Phosphatidylinositol glycan anchor biosynthesis class $\mathrm{T}$ mutation}

Advances in biochemical and genetic analysis are constantly revealing novel causes of recurrent aseptic meningitis. Recently, recurrent aseptic meningitis associated with phosphatidylinositol glycan anchor biosynthesis class T (PIGT) mutation has been reported [39]. A 71-year-old male experienced 121 episodes of recurrent aseptic meningitis over 18 years. Symptoms of meningitis improved spontaneously 7-10 days after onset. Corticosteroid treatment shortened the duration but was not effective in preventing the attacks. Prior to the occurrence of meningitis, he had a history of recurrent urticaria and arthralgia since the age of 30 years. At 68 years, the patient developed severe hemolysis and was diagnosed with paroxysmal nocturnal hemoglobinuria. Genetic analysis revealed no mutations in MEFV, MVK, TNFRSF1A, NLRP3, NLRP12, or $P I G A$ but a germline nonsense mutation (c.250G > T, p.E84X) and a somatic $18 \mathrm{Mbp}$ deletion in the PIGT gene. After administration of eculizumab, an anti-C5 antibody, all symptoms resolved, and the remission has lasted more than 3 years.

\section{Neuronal intranuclear inclusion disease}

Neuronal intranuclear inclusion disease (NIID) is a rare neurodegenerative disease characterized by eosinophilic hyaline intranuclear inclusions in the central, peripheral, and autonomic nervous systems. Since the first case reported in 1968, NIID has been considered a heterogeneous disease due to its highly variable clinical manifestations. Until 2011, about 40 NIID cases, which were diagnosed by postmortem brain biopsy, had been described worldwide. In recent years, skin biopsy and characteristic findings on brain MRI have been used to confirm NIID diagnosis; thus, the number of reported NIID cases has increased. Skin biopsy can detect intranuclear inclusions that are identical, both morphologically and immunohistochemically, to those found in central nervous tissues [40]. In 2019, a GGC repeat expansion in the 5'-UTR of NOTCH2NLC was shown as the genetic cause of NIID [41-43].

The onset age of NIID varies widely from infant to adult. The clinical manifestation of adult-onset NIID can vary, including 
dementia, peripheral neuropathy, autonomic dysfunction, cerebellar ataxia, parkinsonism, seizure, and encephalitis episodes. Dementia is the most prominent symptom in sporadic cases of NIID. The majority of NIID cases have been reported from Japan, with an older age of onset (median, 60 years; interquartile range, 46-66 years) and large proportions of primary cognitive presentation (61\%) and pathognomonic MRI changes (70\%) [44]. Nervous system symptoms are the main manifestations of NIID; however, more than $10 \%$ of patients can present without nervous system symptoms. In a recent study, NIID was shown to present with diverse symptoms of other systems, especially respiratory, digestive, circulatory, and urinary systems [45]. Furthermore, the onset age of initial symptoms in different systems was much younger than that of disease in the nervous system [45].

The typical brain MRI findings are symmetrical leukoencephalopathy accompanied by high signal intensities along the corticomedullary junctions on diffusion-weighted imaging (DWI), termed the "zigzag edging sign." [46]. However, only about $37 \%$ of affected individuals display typical MRI features [47]. Notably, these typical MRI features on DWI are also occasionally observed in fragile $\mathrm{X}$ tremor/ataxia syndrome (FXTAS) caused by mildly expanded CGG repeats in the 5'-UTR of the FMR1 gene and can show similar clinical presentation to NIID. Similar noncoding CGG repeat expansions (in LOC642361/NUTM2B-AS1) were identified in a newly described condition, oculopharyngeal myopathy with leukoencephalopathy, which also can show the typical MRI features [43]. Therefore, diagnostic utility of the zigzag edging sign in various clinical presentations should be further investigated.

The genetic diagnosis of NIID can be challenging, but noncoding CGG repeat expansion can be detected using various techniques. Repeat-primed polymerase chain reaction (PCR), GC-rich PCR, long-read sequencing using nanopore sequencing or single-molecule real-time sequencing, and whole genome sequencing can be utilized for diagnosis of NIID. To date, all confirmed cases of noncoding CGG repeat expansion have been reported in East Asian cohorts. The number of expanded CGG repeats ranged from 66 to 517 in NIID [41] and from 4 to 41 in healthy controls [47]. However, $7.1 \%$ of pathologically confirmed Japanese NIID patients did not contain CGG repeat expansion in NOTCH2NLC [43]. It is not clear whether anticipation occurs in NIID, like in other trinucleotide repeat disorders [45]. However, anticipation was recently observed in familial essential tremor patients with genetically confirmed NIID [47]. Therefore, there is need for further in- vestigations in larger numbers of patients.

In a recent study, none of the 11 pathology-confirmed NIID cases of European ancestry carried the noncoding CGG repeat expansion, indicating that genetically heterogeneous mechanisms underlie NIID in European patients [44]. Therefore, CGG repeat expansions located in other loci or repeat expansions involving similar repeat motifs should be investigated in future studies. Conversely, the GGC repeat expansion in NOTCH2NLC has been identified in a small portion of Alzheimer disease-affected families (1.43\%) and parkinsonism-affected families (1.46\%) [41]. Therefore, NIID should be considered a differential diagnosis for dementia or parkinsonism. Some researchers suggest a concept of NIID-related disorder, which includes NIID and other related neurodegenerative disorders. NIIDs are likely underdiagnosed, and an efficient screening strategy should be established.

Currently, effective treatment to cure or slow the progression of NIID does not exist. Several medications can help improve patient symptoms, but research has been limited due to the small number of confirmed cases. Levodopa responsiveness in cases with parkinsonism is variable and unsustained [48]. In a case report, two NIID patients presented with dopa-responsive juvenile parkinsonism. Both patients showed excellent initial response to levodopa treatment but soon experienced disabling dopa-induced dyskinesia [49]. In another case report, levodopa and trihexyphenidyl were ineffective against tremor in an NIID patient with resting tremor similar to that observed in Parkinson disease [50]. Therefore, levodopa responsiveness in NIID needs to be further investigated. Because NIID can currently be diagnosed using genetic testing, the number of reported NIID cases is rapidly increasing. The effectiveness of various treatments for NIID should be investigated in the near future.

\section{Conclusion}

Diagnosis of AE is increasing, and immunotherapies are delivered to many patients suspected of antibody-negative AEs. In addition, during the last decade, an increasing number of new diseases have been discovered due to diagnostic exome sequencing performed in many rare disease conditions. Differential diagnosis of rare genetic diseases in meningoencephalitis patients is important because it helps exclude the possibility of infection, and certain medications are effective in some cases. Further research on these rare diseases will significantly help these patients. 


\section{Conflicts of Interest}

Jangsup Moon has been an associate editor of encephalitis since October 2020. He was not involved in the review process of this original article. No other potential conflict of interest relevant to this article was reported.

\section{References}

1. Gelfand JM, Genrich G, Green AJ, Tihan T, Cree BA. Encephalitis of unclear origin diagnosed by brain biopsy: a diagnostic challenge. JAMA Neurol 2015;72:66-72.

2. Cellucci T, Van Mater H, Graus F, et al. Clinical approach to the diagnosis of autoimmune encephalitis in the pediatric patient. Neurol Neuroimmunol Neuroinflamm 2020;7:e663.

3. Hermetter C, Fazekas F, Hochmeister S. Systematic review: syndromes, early diagnosis, and treatment in autoimmune encephalitis. Front Neurol 2018;9:706.

4. Mandel-Brehm C, Dubey D, Kryzer TJ, et al. Kelch-like protein 11 antibodies in seminoma-associated paraneoplastic encephalitis. N Engl J Med 2019;381:47-54.

5. Ricken G, Schwaiger C, De Simoni D, et al. Detection methods for autoantibodies in suspected autoimmune encephalitis. Front Neurol 2018;9:841.

6. Honorat JA, Komorowski L, Josephs KA, et al. IgLON5 antibody: neurological accompaniments and outcomes in 20 patients. Neurol Neuroimmunol Neuroinflamm 2017;4:e385.

7. Lee WJ, Lee ST, Byun JI, et al. Rituximab treatment for autoimmune limbic encephalitis in an institutional cohort. Neurology 2016;86: $1683-1691$.

8. Lee WJ, Lee ST, Moon J, et al. Tocilizumab in autoimmune encephalitis refractory to rituximab: an institutional cohort study. Neurotherapeutics 2016;13:824-832.

9. von Rhein B, Wagner J, Widman G, Malter MP, Elger CE, Helmstaedter C. Suspected antibody negative autoimmune limbic encephalitis: outcome of immunotherapy. Acta Neurol Scand 2017;135:134-141.

10. Splinter K, Adams DR, Bacino CA, et al. Effect of genetic diagnosis on patients with previously undiagnosed disease. N Engl J Med 2018;379:2131-2139.

11. Fernandez-Marmiesse A, Gouveia S, Couce ML. NGS technologies as a turning point in rare disease research, diagnosis and treatment. Curr Med Chem 2018;25:404-432.

12. Neokleous A, Psarelis S, Parperis KM. Aseptic Meningitis in hereditary autoinflammatory diseases. Cureus 2020;12:e8244.

13. Borges T, Barbosa A, Silva S. Adult-onset systemic autoinflammatory disorders: a clinical approach. Reumatismo 2020;71:177-188.

14. Gaggiano C, Rigante D, Vitale A, et al. Hints for genetic and clinical differentiation of adult-onset monogenic autoinflammatory diseases. Mediators Inflamm 2019;2019:3293145.

15. Keddie S, Parker T, Lachmann HJ, Ginsberg L. Cryopyrin-associated periodic fever syndrome and the nervous system. Curr Treat Options Neurol 2018;20:43

16. Hoffman HM, Kuemmerle-Deschner JB, Goldbach-Mansky R. Cryopyrin-associated periodic syndromes (CAPS). In: Hashkes PJ, Laxer R, Aimon A, editors. Textbook of autoinflammation. Springer; 2019. p. 347-365.

17. Schuh E, Lohse P, Ertl-Wagner B, et al. Expanding spectrum of neurologic manifestations in patients with NLRP3 low-penetrance mutations. Neurol Neuroimmunol Neuroinflamm 2015;2:e109.

18. Williams R, Hawkins P, Lane T. Recognising and understanding cryopyrin-associated periodic syndrome in adults. Br J Nurs 2019; 28:1180-1186

19. Aksentijevich I, Putnam CD, Remmers EF, et al. The clinical continuum of cryopyrinopathies: novel CIAS1 mutations in North American patients and a new cryopyrin model. Arthritis Rheum 2007;56:12731285.

20. Kuemmerle-Deschner JB, Verma D, Endres T, et al. Clinical and molecular phenotypes of low-penetrance variants of NLRP3: diagnostic and therapeutic challenges. Arthritis Rheumatol 2017;69:2233-2240.

21. Fye KH, Siegel DH, Connolly MK. Diagnosis of Muckle-Wells syndrome: 33 years later. J Rheumatol 2007;34:2505-2506.

22. Kuemmerle-Deschner JB, Ozen S, Tyrrell PN, et al. Diagnostic criteria for cryopyrin-associated periodic syndrome (CAPS). Ann Rheum Dis 2017;76:942-947.

23. Heilig R, Broz P. Function and mechanism of the pyrin inflammasome. Eur J Immunol 2018;48:230-238.

24. Booth DR, Gillmore JD, Lachmann HJ, et al. The genetic basis of autosomal dominant familial Mediterranean fever. QJM 2000;93:217-221.

25. Sohar E, Gafni J, Pras M, Heller H. Familial Mediterranean fever: a survey of 470 cases and review of the literature. Am J Med 1967;43:227253.

26. Feld O, Yahalom G, Livneh A. Neurologic and other systemic manifestations in FMF: published and own experience. Best Pract Res Clin Rheumatol 2012;26:119-133.

27. Hosoi T, Ishii K, Tozaka N, Kishida D, Sekijima Y, Tamaoka A. Familial Mediterranean fever is important in the differential diagnosis of recurrent aseptic Meningitis in Japan. Intern Med 2020;59:125-128.

28. El Roz A, Ghssein G, Khalaf B, Fardoun T, Ibrahim JN. Spectrum of MEFV variants and genotypes among clinically diagnosed FMF patients from Southern Lebanon. Med Sci (Basel) 2020;8:35.

29.Rowczenio DM, Iancu DS, Trojer H, et al. Autosomal dominant familial Mediterranean fever in Northern European Caucasians associated with deletion of p.M694 residue-a case series and genetic exploration. Rheumatology (Oxford) 2017;56:209-213. 
30. Özen S, Batu ED, Demir S. Familial Mediterranean fever: recent developments in pathogenesis and new recommendations for management. Front Immunol 2017;8:253.

31. Savic S, McDermott MF. Tumor necrosis factor (TNF) receptor-associated periodic syndrome (TRAPS). In: Hashkes PJ, Laxer R, Aimon A, editors. Textbook of autoinflammation. Springer; 2019. p. 329-345.

32. Minden K, Aganna E, McDermott MF, Zink A. Tumour necrosis factor receptor associated periodic syndrome (TRAPS) with central nervous system involvement. Ann Rheum Dis 2004;63:1356-1357.

33. Wildemann B, Rudofsky G Jr, Kress B, Jarius S, Konig F, Schwenger V. The tumor-necrosis-factor-associated periodic syndrome, the brain, and tumor-necrosis-factor-alpha antagonists. Neurology 2007;68: $1742-1744$.

34. Haerynck F, Stordeur P, Vandewalle J, et al. Complete factor I deficiency due to dysfunctional factor I with recurrent aseptic meningo-encephalitis. J Clin Immunol 2013;33:1293-1301.

35. Broderick L, Gandhi C, Mueller JL, et al. Mutations of complement factor I and potential mechanisms of neuroinflammation in acute hemorrhagic leukoencephalitis. J Clin Immunol 2013;33:162-171

36. Franco-Jarava C, Álvarez de la Campa E, Solanich X, et al. Early versus late diagnosis of complement factor i deficiency: clinical consequences illustrated in two families with novel homozygous CFI mutations. J Clin Immunol 2017;37:781-789.

37. Nanthapisal S, Eleftheriou D, Gilmour K, et al. Cutaneous vasculitis and recurrent infection caused by deficiency in complement factor I. Front Immunol 2018;9:735.

38. Shields AM, Pagnamenta AT, Pollard AJ, et al. Classical and non-classical presentations of complement factor I deficiency: two contrasting cases diagnosed via genetic and genomic methods. Front Immunol 2019;10:1150.

39. Kawamoto M, Murakami Y, Kinoshita T, Kohara N. Recurrent aseptic meningitis with PIGT mutations: a novel pathogenesis of recurrent meningitis successfully treated by eculizumab. BMJ Case Rep 2018;2018:bcr2018225910.
40. Sone J, Mori K, Inagaki T, et al. Clinicopathological features of adult-onset neuronal intranuclear inclusion disease. Brain 2016; 139(Pt 12):3170-3186.

41. Tian Y, Wang JL, Huang W, et al. Expansion of human-specific GGC repeat in neuronal intranuclear inclusion disease-related disorders. Am J Hum Genet 2019;105:166-176.

42. Sone J, Mitsuhashi S, Fujita A, et al. Long-read sequencing identifies GGC repeat expansions in NOTCH2NLC associated with neuronal intranuclear inclusion disease. Nat Genet 2019;51:1215-1221.

43. Ishiura H, Shibata S, Yoshimura J, et al. Noncoding CGG repeat expansions in neuronal intranuclear inclusion disease, oculopharyngodistal myopathy and an overlapping disease. Nat Genet 2019;51:1222-1232.

44. Chen Z, Yan Yau W, Jaunmuktane Z, et al. Neuronal intranuclear inclusion disease is genetically heterogeneous. Ann Clin Transl Neurol 2020;7:1716-1725.

45. Chen H, Lu L, Wang B, et al. Re-defining the clinicopathological spectrum of neuronal intranuclear inclusion disease. Ann Clin Transl Neurol 2020;7:1930-1941.

46. Chen L, Chen A, Lei S, He L, Zhou M. Teaching NeuroImages: the zigzag edging sign of adult-onset neuronal intranuclear inclusion disease. Neurology 2019;92:e2295-e2296.

47. Sun QY, Xu Q, Tian Y, et al. Expansion of GGC repeat in the human-specific NOTCH2NLC gene is associated with essential tremor. Brain 2020;143:222-233.

48. Paviour DC, Revesz T, Holton JL, Evans A, Olsson JE, Lees AJ. Neuronal intranuclear inclusion disease: report on a case originally diagnosed as dopa-responsive dystonia with Lewy bodies. Mov Disord 2005;20:1345-1349.

49. Lai SC, Jung SM, Grattan-Smith P, et al. Neuronal intranuclear inclusion disease: two cases of dopa-responsive juvenile parkinsonism with drug-induced dyskinesia. Mov Disord 2010;25:1274-1279.

50. Kitagawa N, Sone J, Sobue G, Kuroda M, Sakurai M. Neuronal intranuclear inclusion disease presenting with resting tremor. Case Rep Neurol 2014;6:176-180. 